\title{
Anamorphic zoom system based on liquid crystal displays
}

Claudio Iemmi

iemmi@df.uba.ar

Juan Campos

juan.campos@uab.es
Departamento de Física, Facultad de Ciencias Exactas y Naturales, Universidad de Buenos Aires, 1428 Buenos Aires, Argentina

Departamento de Física, Universidad Autónoma de Barcelona, 08193 Bellaterra, Spain

In this work we present an anamorphic zoom system, based on liquid crystal displays, which allows changing the magnification and distortion of an image very quickly, without mechanical parts and keeping the output plane stationary. The anamorphic lenses that make up the optical processor are obtained by displaying a combination of convergent-divergent cylindrical lenses, with arbitrary orientations, onto the spatial light modulators. In order to illustrate the capabilities of the system, some experimental results are shown. [DOI: $10.2971 /$ jeos.2009.09029]

Keywords: active optics, spatial light modulators, liquid crystals, zoom systems, anamorphic systems

\section{INTRODUCTION}

Optical systems containing active elements are playing an increasing role in different areas. Besides the well known systems used to improve the image quality in telescopes there are a variety of applications, such us optical interconnections [1], beam steering [2], metrology [3, 4], image processing [5][7], etc., in which active optics are employed. Usually these devices are lighter, smaller and more flexible than those that use conventional optical elements; moreover they can be reconfigured very quickly.

Active optical elements have found a widespread use in imaging systems, they are capable of focusing, zooming and scanning without mechanical moving parts. In traditional technologies, where lenses of fixed focal lengths are employed, these operations are implemented by changing the distances between lenses, the object and the image plane. Alternatively it is possible to vary the focusing power of a lens by changing its shape or its refractive index. Recently miniaturized systems, based on fluidic lenses, have been developed. In these cases the shape of the lens is changed by applying an electric field. These devices are mainly designed to be used in camera phones [8] or as compensators of astigmatic laser beams [9]. Lenses made of birefringent liquid crystal change their refractive index when an electric field is applied [10] but, good quality images and rapid responses are only obtained for very thin layers of liquid crystal. As thin layers lead to small optical path changes, it is necessary to display Fresnel type lenses onto pixilated structures in order to implement an active imaging system with good performance. Active optics based on programmable spatial light modulators (SLMs) has been advantageously used in zoom systems [11, 12] giving place to devices reduced in size, weight and with the possibility to vary magnification faster than mechanical zooms. It is true that unwanted diffracted orders are originated by the pix- elated structure, nevertheless by using programmable SLMs it is possible to address pixel to pixel arbitrary phase distributions such as multiplexed or anamorphic lenses [13]-[16] that give place to more flexible designs.

In this paper we combine, in an active optic system, zooming capability with anamorphosis. Many optical processors require these properties in order to carry out different operations: data elaboration from synthetic aperture radars [17], map transformation [18] and fractional Fourier transform [19] are some examples. In the next section we describe the proposed optical system and we show the obtained experimental results.

\section{DESCRIPTION OF THE SYSTEM AND EXPERIMENTAL RESULTS}

A scheme of the experimental set up is shown in Figure 1. Light from an Ar laser (458 $\mathrm{nm}$ line) impinges onto a rotating ground glass diffuser $D$ used in order to diminish the spatial coherence of the source. The object $O$, which is uniformly illuminated by means of condenser $C$, is placed at focal distance $f=135 \mathrm{~nm}$ from lens $L_{1}$, in this way the object is imaged at infinite. Next a telescope, where two spatial light modulators play the role of variable focus anamorphic lenses, constitutes the central part of the proposed system and is able to provide directional angular magnification.

Each anamorphic lens is a diffractive optic programmable element and is conformed by a set of polarizer $P$ and wave plate $W$ in front of a liquid crystal display (LCD), and an analogous set behind it. These polarization elements together with the LCD made up a spatial light modulator that provides phase only modulation when the optimization procedure proposed 


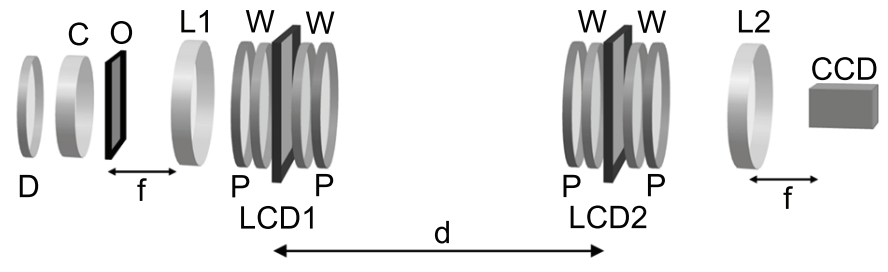

FIC. 1 Sketch of the anamorphic zoom system. $D$ is a diffuser, $C$ a condenser lens, $O$ the object, $L_{1}$ and $L_{2}$ are lenses, $W s$ and $P S$ are waveplates and polarizers respectively, $L C D_{1}$ and $L C D_{2}$ are liquid crystal displays and $C C D$ is a detector.

in [20] is followed. LCD1 and LCD2 are Sony liquid crystal panels, model LCX012BL, extracted from a commercial videoprojector. These displays can work at video rate (30 fps) and their spatial resolution is $640 \times 480$ pixels, with a pixel width of $34 \mu \mathrm{m}$ and a center to center distance of $41 \mu \mathrm{m}$. The phase shift dynamic range is close to $360^{\circ}$ at $458 \mathrm{~nm}$ and the amplitude modulation is almost flat.

Diffraction efficiency of Fresnel lenses, displayed on LCDs, can be reduced basically by three factors [21]: The transmission of the display for the operating wavelength, which depends on the reflection, absorption and fill factor. Moreover, since the display usually works between polarizers and waveplates, it is also affected by reflections and absorption at these elements, being in this case dependent on their particular configuration. Other factor is the fraction of light going to the zero diffraction order generated by the pixelated grid structure. This parameter depends on the fill factor of the display, and for our device we measured a value around $47 \%$. The third factor is the modulation diffraction efficiency, which depends on the complex modulation provided by the display. A deep study about this topic was done by Márquez et. al [22]. Also the inhomogeneities of the display may affect the image quality [23].

As we previously said the proposed system is basically a telescope, so the distance $d$ between LCDs remains fixed and was chosen to equal the sum of the focal distances of the lenses displayed onto the modulators. Let us call $f_{L C D 1}$ and $f_{L C D 2}$ these focal lengths, then it is possible to change simultaneously their value in such a way that $d=f_{L C D 1}+f_{L C D 2}$ remains constant but the magnification $m=f_{L C D 1} / f_{L C D 2}$ changes. In order to achieve large zooming capability, without varying the lens spacing, it is necessary that the active element possess a wide focal-distance tuning range and that the programmed lenses can be either positive or negative. It is well known that the sampling of a Fresnel lens causes the appearance of multiple lenses when the sampling frequency is lower than the Nyquist limit. To avoid this effect we design lenses with focal lengths larger than $|100| \mathrm{cm}$.

Finally the parallel beam of light emerging from the telescope system is focused by lens $L_{2}(f=135 \mathrm{~mm})$ onto a charge coupled device (CCD) placed in its focal plane, in such a way that a modified image of the object can be registered at a fixed position.

It should be pointed out that the multiple orders generated by the pixilated structure of the LCDs perturb the image quality. In order to reduce this effect a diaphragm, not included in the sketch, is used to limit the size of the object.

We can express the phase factor introduced by an anamorphic lens as that produced by a combination of two cylindrical lenses in contact

$$
\begin{aligned}
\Phi\left(x, y, \theta_{1}, \theta_{2}, f_{1}, f_{2}\right)= & -\frac{\pi}{\lambda f_{1}}\left(x \cos \theta_{1}+y \sin \theta_{1}\right)^{2} \\
& -\frac{\pi}{\lambda f_{2}}\left(x \cos \theta_{2}+y \sin \theta_{2}\right)^{2},
\end{aligned}
$$

where $\lambda$ is the wavelength, $f_{1}$ and $f_{2}$ are the focal lengths of the cylindrical lenses and $\theta_{1}$ and $\theta_{2}$ are the angles between the axes of the lenses and the vertical of the laboratory. It is easy to see that if $\theta_{1}=0^{\circ}, \theta_{2}=90^{\circ}$ and $f_{1}=f_{2}$ we recover the expression for a spherical lens. The focal lengths can be either positive or negative, nevertheless, in order to shorten the distance between LCDs it is convenient to choose a combination with a positive $f$ value for the lens displayed onto one modulator and a negative value for the other. The flexibility on the combination of convergent and divergent lenses allows magnifications, demagnifications and distortions on the final image.

With the purpose of illustrating the capabilities of the proposed system we performed some experiences using as object an AF target. In first place, combinations of spherical lenses were displayed onto the modulators in order to obtain images with different magnifications.

As an example, in Figure 2 images of the target with a zoom factor of about 2 are shown. Figure 2(a) corresponds to the case when no lens is programmed onto the LCDs. In this situation the object is imaged onto the CCD by the system conformed by the conventional lenses $L_{1}$ and $L_{2}$ as object and detector are placed in conjugated planes. In order to obtain the image shown in Figure 2(b) we chose $f_{L C D 1}=300 \mathrm{~cm}$ and $f_{L C D 2}=-150 \mathrm{~cm}$.

These features are maintained when controlled distortions are introduced by means of anamorphic lenses.

In Figure 3 three different effects are shown. For the horizontal stretching shown in Figure 3(a), cylindrical lenses with focal distances $300 \mathrm{~cm}$ and $-150 \mathrm{~cm}$ where displayed on LCD1
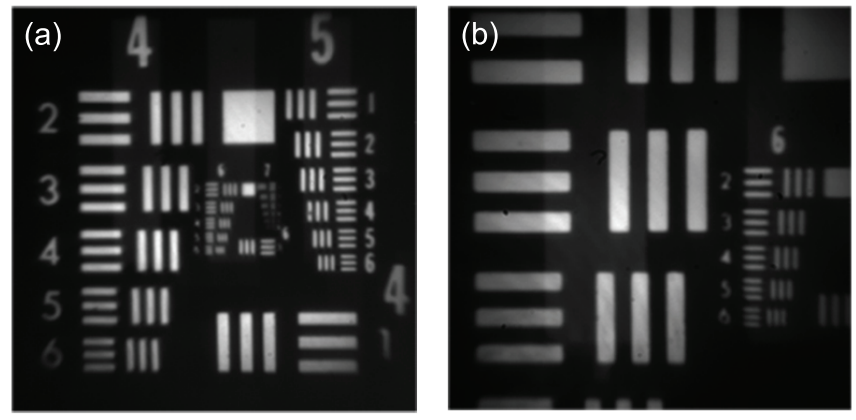

FIG. 2 Two images obtained by displaying onto the SLMs spherical lenses with variable focal length. The zoom factor between a) and b) is approximately 2 . 

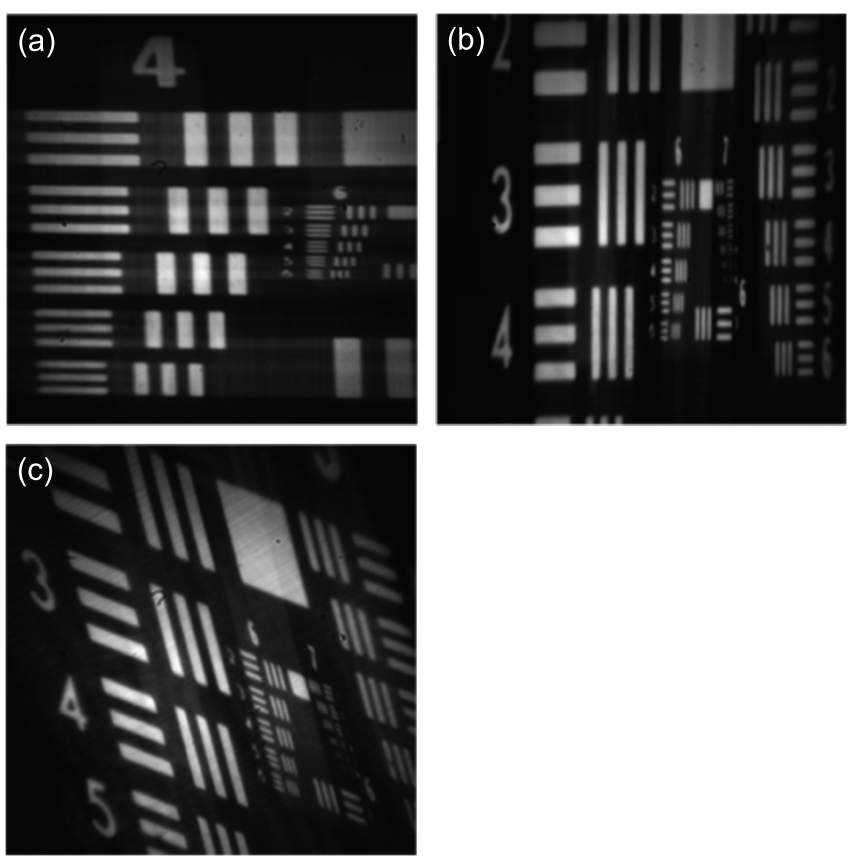

FIG. 3 Distortions introduced by means of the anomorphic lenses: a) horizontal stretching, b) vertical stretching and c) diagonal stretching.

and $L C D 2$ respectively. In this way a magnification of 2 is obtained in the horizontal direction and a magnification of 1 in the vertical direction. The same but in the orthogonal direction was done in order to obtain the vertical stretching shown in Figure 3(b). Finally an arbitrary deformation was obtained by programming two orthogonal pairs of cylindrical lenses. Each pair must fulfil the condition that the sum of their focal distances equals $d$. The one oriented at $30^{\circ}$, with respect to the vertical of the laboratory, has a higher magnification than the oriented at $120^{\circ}$. The final effect is a diagonal stretching of the image at $120^{\circ}$.

In all cases, besides the faint perturbation originated by the pixilated structure of the LCD it is noticeable the good quality of the images and the preservation of the final plane position.

\section{CONCLUSIONS}

In this paper we have proposed an active anamorphic optical system with zooming capabilities. The device is very flexible and can be reconfigured at video rate without using mechanical displacements as it is based on liquid crystal display technology. The anamorphosis is achieved by programming combinations of quadratic phases with arbitrary orientations onto the spatial light modulators. Very good quality images are obtained for a large range of zoom ratios and distortion factors.

\section{ACKNOWLEDGEMENTS}

We acknowledge financial support from Spanish Ministerio de Educación y Ciencia (FIS2006-13037-C02-01 and 02). C. Iemmi acknowledges support from Univ. Buenos Aires and CONICET (Argentina) and Generalitat de Catalunya (2006PIV00011).

\section{References}

[1] I. Fujieda, 0. Mikami, and A. Ozawa "Active Optical Interconnect Based on Liquid-Crystal Grating" Appl. Optics 42, 1520-1525 (2003).

[2] M. Capeluto, C. LaMela, C. Iemmi, and M. Marconi "Scanning mechanism based on a programmable liquid crystal display" Opt. Commun. 232, 107-113 (2004).

[3] J. Kacperski and M. Kujawinska "Active, LCoS based laser interferometer for microelements studies" 0pt. Express 14, 9664-9678 (2006).

[4] C. Iemmi, A. Moreno, J. Nicolás, and J. Campos "Evaluation and correction of aberrations in an optical correlator by phase shift interferometry" Opt. Lett. 28, 1117-1119 (2003).

[5] I. Moreno, J. Davis, and K. Crabtree "Fractional Fourier transform optical system with programmable diffractive lenses" Appl. Optics 42, 6544-6548 (2003).

[6] C. Iemmi, A. Moreno, and J. Campos "Digital holography with a point diffraction interferometer" Opt. Express 13, 1885-1891 (2005).

[7] C. La Mela and C. Iemmi "Optical encryption using phase-shifting interferometry in a joint transform correlator" Opt. Lett. 31, 25622564 (2006).

[8] S. Kuiper and B. H. W. Hendriks "Variable-focus liquid lens for miniature cameras" Appl. Phys. Lett. 85, 1128-1130 (2004).

[9] T. L. Kelly, A. F. Naumov, M. Yu. Loktev, M. A. Rakhmatulin, and 0 . A. Zayakin "Focusing of astigmatic laser diode beam by combination of adaptive liquid crystal lenses" Opt. Commun. 181, 295301 (2000).

[10] H. Ren, D. W. Fox, B. Wu, and S. T. Wu, "Liquid crystal lens with large focal length tunability and low operating voltage" Opt. Express 15, 11328-11335 (2007).

[11] E. C. Tam "Smart electro-optical zoom lens" Opt. Lett. 17, 369-371 (1992).

[12] D. V. Wick and T. Martinez “Adaptive optical zoom" Opt. Eng. 43, 8-9 (2004).

[13] A. Márquez, C. Iemmi, J. Campos, and M. J. Yzuel "Achromatic diffractive lens onto a liquid cristal display" Opt. Lett. 31, 392-394 (2006).

[14] C. Iemmi, J. Campos, J. Escalera, 0. López-Coronado, R. Gimeno, and M. Yzuel "Depth of focus increase by multiplexing programmable diffractive lenses" Opt. Express 14, 10207-10219 (2006).

[15] J. A. Davis, H. M. Schley-Seebold, and D. Cottrell "Anamorphic optical systems using programmable spatial light modulators" Appl. Optics 31, 6185-6186 (1992).

[16] M. S. Millán, J. Otón, and E. Pérez-Cabré “Chromatic compensation of programmable Fresnel lenses" Opt. Express 14, 6226-6242 (2006).

[17] A. Kozma, E. N. Leith, and N. G. Massey "Tilted plane optical processor" Appl. Optics 11, 1766-1776 (1972).

[18] D. Wang, A. Pe'er, A. A. Friesem, and A. W. Lohmann "General linear optical coordinate transformations" J. Opt. Soc. Am. A 17, 1864-1869 (2000).

[19] I. Moreno, C. Ferreira, and M. Sánchez-Lopez "Ray matrix analysis of anamorphic fractional Fourier systems" J. Opt. A-Pure Appl. Op. 8, 427-435 (2006).

[20] A. Márquez, C. Iemmi, I. Moreno, J. A. Davis, J. Campos, and M. J. Yzuel "Quantitative prediction of the modulation behavior 
of twisted nematic liquid crystal displays based on a simple physical model" Opt. Eng. 40, 2558-2564 (2001).

[21] I. Moreno, C. Iemmi, A. Márquez, J. Campos, and M. J. Yzuel “Modulation light efficiency of diffractive lenses displayed onto a restricted phase-mostly modulation display" Appl. Optics 43, 62786284 (2004).

[22] A. Márquez, C. Iemmi, I. Moreno, J. Campos, and M. J. Yzuel
"Anamorphic and spatial frequency dependent phase modulation on liquid crystal displays. Optimization of the modulation diffraction efficiency" Opt. Express 13, 2111-2119 (2005).

[23] J. Otón, P. Ambs, M. S. Millán, and E. Pérez-Cabré “Multipoint phase calibration for improved compensation of inherent wavefront distortion in parallel aligned liquid crystal on silicon displays" Appl. Optics 46, 5667-5679 (2007). 